\title{
Article original / Original article Expérimentation de la culture de chanvre industriel à fibres au Maroc
}

\section{Experiment of industrial hemp crops in Morocco}

\author{
Hamid Stambouli $^{1 \star}$, Aziz El Bouri ${ }^{1}$, Taoufiq Bouayoun ${ }^{1}$, Naima El Karni ${ }^{1}$, Z. Naciri ${ }^{1}$, Aziz Johar ${ }^{1}$, \\ Amal Saoura ${ }^{1}$, Seddik Saidi ${ }^{2}$ \\ ${ }^{1}$ Laboratoire de Recherches et d'Analyses Techniques et Scientifiques - LARATES, Gendarmerie Royale, BP 6597, Rabat-Instituts CP \\ 10100 Rabat, Maroc \\ 2 Institut National de Recherche Agronomique - INRA, Avenue Annasr, BP 415 RP Rabat, Maroc
}

\begin{abstract}
Résumé - Objectif : Dans la perspective de la légalisation de la culture de chanvre à fibres au Maroc, une expérimentation de cette culture a été réalisée entre le 19/04/2010 et le 10/08/2010 dans quatre régions différentes du pays, et a concerné les trois variétés Santhica 27, Epsilon 68 et Futura 75, toutes autorisées en Europe $([\Delta-9-\mathrm{THC}]<0.2 \%)$. Méthodes : Les essais ayant été conduits dans des régions de situations géographiques, conditions climatiques et caractéristiques pédologiques différentes des cultures européennes, il convenait alors de vérifier le comportement de ces plantes par le contrôle des rendements agronomiques et de leur teneur en $\Delta-9$-THC. Les protocoles d'échantillonnage, de séchage et d'évaluation du taux du $\triangle-9-$ THC au sein de ces cultures, ont d'abord été réalisés d'une part conformément à la procédure B de l'Arrêté du 24 février 2004 du Règlement CE N 1177/2000, et ensuite selon une méthode simplifiée basée sur la détermination du rapport $\alpha=\Delta-9-\mathrm{THC} / \mathrm{CBD}$, d'autre part. Résultats : Les meilleurs rendements en matière sèche, densité et hauteurs des plantes sont obtenus avec la variété Futura 75 dans les quatre sites, suivie de Epsilon 68 et de Santhica 27. Le contrôle des teneurs de ces cultures en $\Delta-9$-THC a conduit à des taux moyens inférieurs à la limite autorisée puisqu'ils se situent entre $0,013 \%$ et $0,027 \%$ pour la variété Epsilon 68 , entre $0,023 \%$ et $0,035 \%$ pour la variété Futura 75 et des taux quasiment nuls (non détectables par GCFID) pour la variété Santhica 27. L'analyse de cette dernière variété par GC/MS mode SIM et en LCMSMS a permis la mise en évidence de traces du $\Delta$-9-THC. Par ailleurs, le rapport $\alpha$ évalué respectivement à 0,042 et 0,047 pour les deux variétés Epsilon 68 et Futura 75 , est de même ordre de grandeur que les valeurs obtenues habituellement pour les cultures de chanvre à fibres et reste nettement inférieur à 0,2. Conclusion : Les résultats préliminaires obtenus par les deux procédures réglementaire et simplifiée concordent et ne révèlent aucun dépassement des valeurs limites pour les trois variétés testées bien que les conditions de culture ne soient pas rigoureusement les mêmes que celles adoptées en Europe. En outre, la photopériode qui caractérise ces régions du sud de la Méditerranée étant très favorable, elle s'est traduite par une maturité rapide des cultures qui au bout de deux mois et demi ont toutes atteint le stade de fin de floraison.
\end{abstract}

Mots clés : Chanvre industriel, culture, $\Delta$-9-tetrahydrocannabinol, cannabidiol, réglementation.

\begin{abstract}
Objectives: In anticipation of the legalization of hemp fiber cultivation in Morocco, an experiment of this crops was conducted between 19/04/2010 and 10/08/2010 in four different regions of the country, and concerned three varieties Santhica 27, Epsilon 68 and Futura 75, all authorized in Europe $([\Delta-9-\mathrm{THC}]<0.2 \%)$. Methods: The tests were conducted in areas with geographical, climatic and soil characteristics different than European cultures, that's why it was necessary to verify the behavior of these plants by controlling their $\Delta$-9-THC content. Sampling, drying and $[\Delta-9-\mathrm{THC}]$ evaluation in these crops were performed according to procedure $\mathrm{B}$ of European Regulation EC $\mathrm{N}^{\circ}$ 1177/2000, and using a simplified method based on the determination of the ratio $\alpha=\Delta-9$-THC/CBD. Results: The best dry matter yields, plant height and density are obtained with the variety Futura 75 in the four sites, followed by Epsilon 68 and Santhica 27. The average contents of these crops in $\Delta-9-$ THC were lower than the limit of $0.2 \%$ and ranged from $0.013 \%$ to $0.027 \%$ for the variety Epsilon 68, between $0.023 \%$ and $0.035 \%$ for the variety Futura 75
\end{abstract}

\footnotetext{
^ Correspondance :

Hamid Stambouli, Tél. et Fax +212 (0) 5376425 04,

labgr@menara.ma
} 
and null (undetectable by GCFID) for variety Santhica 27. Analysis of this latter variety by GC / MS SIM mode and LCMSMS allowed the identification of traces of $\Delta$-9-THC. Similarly, the ratio $\alpha$ has been evaluated to 0.042 and 0.047 respectively for Epsilon 68 and Futura 75 varieties which is similar to those usually obtained for hemp fiber crops and stays muc hlower than 0.2. Conclusion: The preliminary results obtained by both methods reveal no exceeded values however the culture conditions are not exactly the same as those adopted in Europe. In addition, photoperiod characteristic of these south Mediterranean regions are very favourable, thereby resulting in a fast maturing crops which have all reached the late stage of flowering after two and half months.

Key words: Industrial hemp, crops, $\Delta$-9-tetrahydrocannabinol, cannabidiol, regulation.

Reçu le 3 février 2011, accepté après modifications le 31 mars 2011

Publication en ligne le 15 juin 2011

\section{Introduction}

La culture du Cannabis est connue depuis fort longtemps. La plante se présente sous deux principales sous-espèces : le Cannabis sativa L. subsp. indica ou chanvre indien, recherché principalement pour les propriétés psychotropes de son principe actif le $\Delta$-9-tetrahydrocannabinol « $\Delta$-9-THC» et le Cannabis sativa L. subsp. sativa ou chanvre industriel, pauvre en THC et très réputé pour ses graines, fibres et chènevottes [1]. En Europe, la culture de certaines variétés de cannabis dont le taux de $\Delta-9-$ THC ne dépasse pas $0,2 \%$ est autorisée pour des utilisations industrielles réglementées (textile, isolant, plasturgie, matériaux de construction, cosmétique, alimentation...) et représente chaque année de 15000 à 18000 ha [2]. La France et la Chine en sont les premiers producteurs respectivement au niveau européen et mondial. D'après la $\mathrm{FAO}$, la production mondiale du chanvre à fibres est passée de 50000 t en 2000 à 90000 t en 2005 [3]. La production européenne de la fibre a atteint 35000 t pour la seule année 2009, avec comme principal débouché l'industrie papetière, alors que pour la chènevotte, deuxième constituant majoritaire de la tige, elle est évaluée à 60000 t, exploitée essentiellement dans l'industrie du bâtiment [4].

L'intérêt de plus en plus croissant que suscite la culture du chanvre à fibres est motivé par des raisons écologiques et économiques. En effet, cette culture ne nécessite pas d'utilisation de produits phytosanitaires et est très économe en intrants. L'exploitation de la fibre de la plante dans la production du papier peut contribuer à la lutte contre la déforestation et le déboisement, et les applications de sa chènevotte dans l'industrie du bâtiment comme matériau d'isolation des toitures et des murs constituent une alternative écologique à l'amiante et à la laine de verre. La chènevotte est également retrouvée dans l'industrie du béton et en plasturgie automobile.

De même, le chènevis, fruit du chanvre, est une graine oléagineuse qui contient en moyenne $25 \%$ d'acides gras, $25 \%$ de protéines, $30 \%$ de glucides, $15 \%$ de fibres insolubles, carotène, oligoéléments (potassium, magnésium, soufre, calcium, fer et zinc), vitamines (E, C, B1, B2, B3 et B6) et trouve des applications en oisellerie, pêche, alimentation humaine et cosmétique.

Depuis la légalisation des cultures du chanvre à fibres par certains pays, la recherche s'est orientée vers la sélection de variétés de plus en plus pauvres en cannabinoïdes (tableau I), ainsi aux premières générations (Epsilon 68, Futura 75, etc.) qui renfermaient encore des traces de THC, se sont succédé les deuxièmes et troisièmes générations quasiment exemptes de cannabinoïdes. Des listes de variétés autorisées lists of approved cultivars sont alors établies et toute variété qui présente une teneur supérieure au seuil $(0,2 \%)$ de $\Delta$-9-THC fixé par la réglementation est retirée de la liste. Les cultures qui utilisent des semences certifiées des variétés inscrites à l'annexe $\mathrm{V}$ du règlement CE-2860/2000, sous réserve de respecter les autres contraintes réglementaires, peuvent prétendre à une aide financière de la communauté européenne.

$\mathrm{Au}$ Maroc, si les premières cultures du cannabis type drogue remontent jusqu' au $\mathrm{VI}^{\mathrm{e}}$ siècle, celle du chanvre à fibres est peu connue, puisque les premiers essais furent pratiqués avant l'adoption de la Convention unique sur les stupéfiants par les Nations unies en 1961. C'est pourquoi nous nous sommes intéressés en 2010 à l'expérimentation de trois variétés de chanvre industriel Epsilon 68, Futura 75 et Santhica 27, autorisées en Europe mais jamais cultivées dans les conditions climatiques dans cette région d'Afrique du Nord. À cet effet, des essais ont été conduits dans les domaines expérimentaux de l'Institut National de Recherche Agronomique (INRA) situés dans quatre régions du pays (figure 1) :

- Région Agadir, au Sud-Ouest du littoral atlantique (altitude $1250 \mathrm{~m}$ ), caractérisée par un sol sablonneux et une pluviométrie moyenne de $250 \mathrm{~mm} / \mathrm{an}$.

- Région Sefrou, au Moyen Atlas (altitude 850 m), connue pour son sol caillouteux et une pluviométrie moyenne de $640 \mathrm{~mm} / \mathrm{an}$.

- Région Allal Tazi, au Nord-Ouest (altitude $40 \mathrm{~m}$ ), ayant un sol caillouteux/sablonneux et une pluviométrie moyenne de $460 \mathrm{~mm} / \mathrm{an}$.

- Région Béni-Mellal, au centre du pays, porte du Moyen Atlas (altitude $2000 \mathrm{~m}$ ), caractérisée par un sol argileux et une pluviométrie moyenne de 600 à 800 mm/an.

\section{Matériels et méthodes}

Les variétés concernées par cette étude ont été fournies par la Fédération Nationale des Producteurs de Chanvre FNPC France. Il s'agit de trois variétés de chanvre industriel, de type monoïque, inscrites aux catalogues français et européens, Santhica 27, Futura 75 et Epsilon 68 (tableau II). Les trois variétés ont été cultivées sur 12 parcelles de superficie unitaire de $2500 \mathrm{~m}^{2}$. Les dates de semence s'étendent du 19 avril au 28 mai 2010 et celles de la fin de floraison du 16 juin au 3 août 2010 (tableau III). La dose de semis a été déterminée selon le poids de mille graines et la faculté de germination de manière à obtenir une densité de 300 plantes $/ \mathrm{m}^{2}$. 


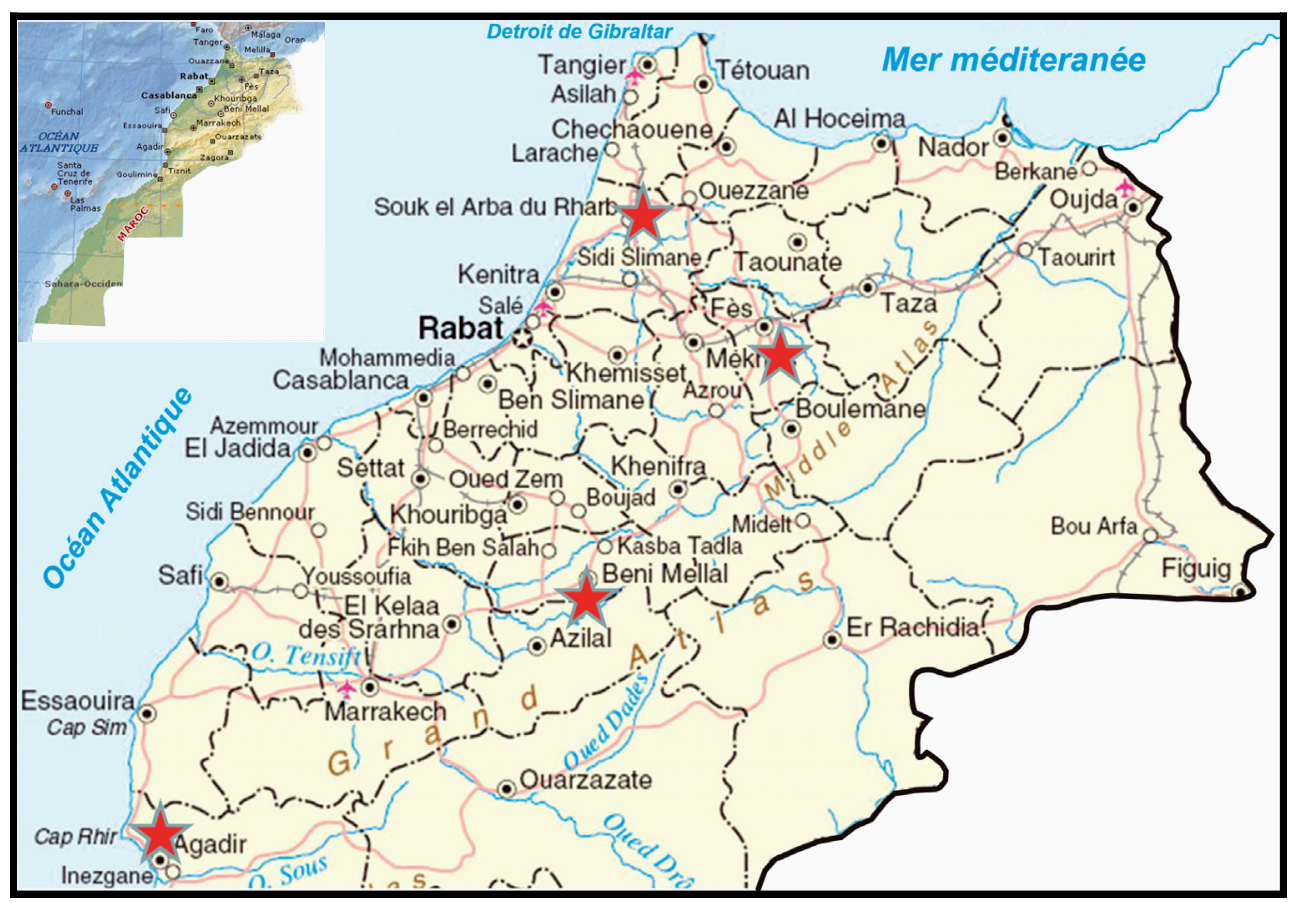

Fig. 1. Localisation géographique des sites d'expérimentation de la culture du chanvre industriel.

Tableau I. Proportions des principaux cannabinoïdes présents dans divers chimiotypes du chanvre [5].

\begin{tabular}{|c|c|c|c|c|c|}
\hline Cannabinoïdes & Drogues & Drogues intermédiaires & \multicolumn{3}{|c|}{ Fibres } \\
\cline { 3 - 6 } & & & $\begin{array}{c}1^{\text {re }} \\
(\text { Epsilon, Futura, } \\
\text { Fedora...) }\end{array}$ & $\begin{array}{c}2^{\mathrm{e}} \\
\text { génération } \\
\text { génération }\end{array}$ & $\begin{array}{c}3^{\mathrm{e}} \\
\text { génération } \\
\text { Santhica }\end{array}$ \\
\hline$\Delta$-9-THC & ++++ & +++ & $+(<0,2 \%)$ & - & - \\
\hline CBD & \pm & +++ & +++ & \pm & - \\
\hline CBG & \pm & \pm & \pm & +++ & - \\
\hline
\end{tabular}

Tableau II. Variétés de chanvre industriel testées.

\begin{tabular}{|c|c|c|c|c|}
\hline Variété & Année d'inscription & $\Delta$-9-THC (\%) & PMG (en g) & Dose de semis (kg/ha) \\
\hline Santhica 27 & 2002 & $<0,001$ & 16,8 & 50 \\
\hline Futura 75 & 1998 & 0,06 & 18,9 & 58 \\
\hline Epsilon 68 & 1996 & 0,04 & 19,3 & 57 \\
\hline
\end{tabular}

PMG : poids de mille graines.

Tableau III. Récapitulatif des essais de culture de chanvre industriel.

\begin{tabular}{|c|c|c|c|c|c|}
\hline Région & Variété & Date de semis & Début de floraison & Fin de floraison & Date de prélèvement \\
\hline \multirow{3}{*}{ Agadir } & Santhica 27 & \multirow{3}{*}{ 19/04/2010 } & $07 / 06 / 2010$ & $16 / 06 / 2010$ & \multirow{3}{*}{ 22/06/2010 } \\
\hline & Epsilon 68 & & $07 / 06 / 2010$ & $18 / 06 / 2010$ & \\
\hline & Futura 75 & & $09 / 06 / 2010$ & $18 / 06 / 2010$ & \\
\hline \multirow{3}{*}{ Béni-Mellal } & Santhica 27 & \multirow{3}{*}{$11 / 05 / 2010$} & $04 / 07 / 2010$ & $13 / 07 / 2010$ & \multirow{3}{*}{$15 / 07 / 2010$} \\
\hline & Epsilon 68 & & $02 / 07 / 2010$ & $13 / 07 / 2010$ & \\
\hline & Futura 75 & & $04 / 07 / 2010$ & $13 / 07 / 2010$ & \\
\hline \multirow{3}{*}{ Allal Tazi } & Santhica 27 & \multirow{3}{*}{$17 / 05 / 2010$} & $23 / 06 / 2010$ & $09 / 07 / 2010$ & \multirow{3}{*}{ 20/07/2010 } \\
\hline & Epsilon 68 & & $24 / 06 / 2010$ & $13 / 07 / 2010$ & \\
\hline & Futura 75 & & $25 / 06 / 2010$ & $16 / 07 / 2010$ & \\
\hline \multirow{3}{*}{ Sefrou } & Santhica 27 & \multirow{3}{*}{$28 / 05 / 2010$} & $14 / 07 / 2010$ & $03 / 08 / 2010$ & \multirow{3}{*}{$10 / 08 / 2010$} \\
\hline & Epsilon 68 & & $14 / 07 / 2010$ & $03 / 08 / 2010$ & \\
\hline & Futura 75 & & $14 / 07 / 2010$ & $03 / 08 / 2010$ & \\
\hline
\end{tabular}


Tableau IV. Récapitulatif des rendements agronomiques.

\begin{tabular}{|c|c|c|c|c|c|c|c|c|c|c|c|c|}
\hline \multirow[b]{2}{*}{ Variété } & \multicolumn{4}{|c|}{ Densité (nb plantes $/ \mathrm{m}^{2}$ ) } & \multicolumn{4}{|c|}{ Rdt matière sèche $(\mathrm{t} / \mathrm{ha})$} & \multicolumn{4}{|c|}{ Hauteurs moyennes des plantes $(\mathrm{m})$} \\
\hline & 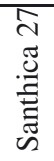 & 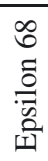 & 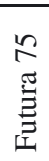 & 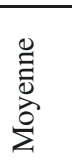 & 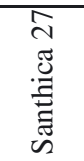 & 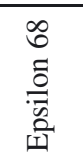 & 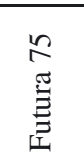 & 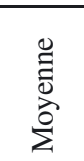 & 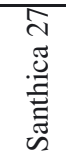 & 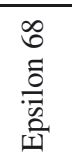 & 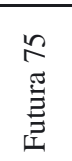 & 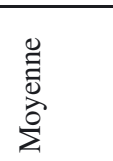 \\
\hline Béni-Mellal & 48 & 57 & 75 & 60 & 16,33 & 21,33 & 24,50 & 20,72 & 1,63 & 2,03 & 2,27 & 1,97 \\
\hline Agadir & 45 & 51 & 64 & 53 & 5,45 & 7,02 & 9,92 & 7,46 & 1,14 & 1,13 & 1,48 & 1,23 \\
\hline Allal Tazi & 35 & 30 & 63 & 43 & 8,33 & 11,00 & 13,3 & 10,88 & 1,66 & 1,77 & 1,90 & 1,78 \\
\hline Sefrou & 35 & 38 & 42 & 38 & 4,47 & 5,34 & 5,58 & 5,13 & 1,45 & 1,38 & 1,52 & 1,45 \\
\hline
\end{tabular}

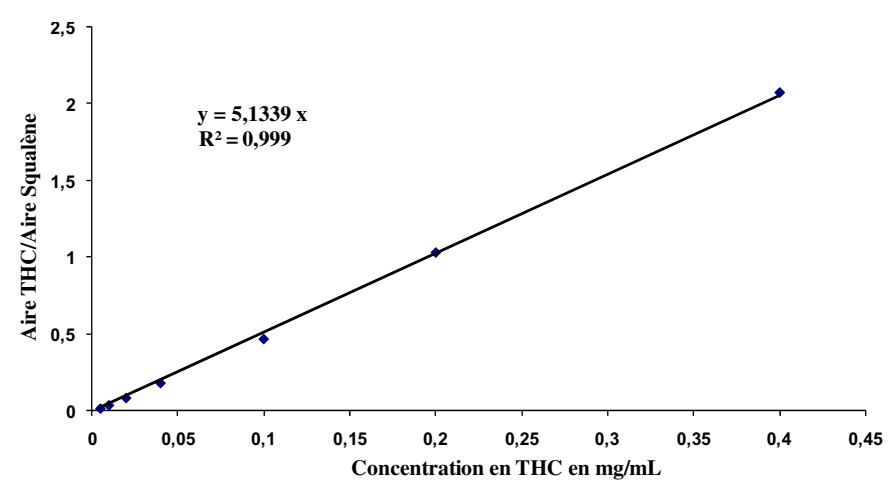

Fig. 2. Droite d'étalonnage du dosage du $\Delta$-9-THC.

Le contrôle de ces cultures a été réalisé par deux méthodes différentes d'abord conformément à la procédure $\mathrm{B}$ du règlement européen $C E N^{\circ} 1177 / 2000$ [6] qui préconise un échantillonnage des tiers supérieurs de 200 plantes/parcelle en vue de l'évaluation de leur teneur en $\Delta-9$-THC par étalonnage externe et ensuite selon la méthode proposée par Fournier et coll. [7,8] consistant en la détermination du rapport $\alpha$ $\Delta-9-\mathrm{THC} / \mathrm{CBD}$ à partir d'un échantillonnage de 200 feuilles exclusivement. Ce rapport ne doit pas excéder 0,2. Cette procédure allège considérablement le contrôle par la réduction du volume du matériel végétal, et par la détermination chromatographique du rapport $\alpha$ à partir des aires des pics sans recours à la gamme d'étalonnage, ce qui est moins contraignant.

Dans les deux cas, l'échantillonnage est réalisé dix jours après la fin de la floraison, pendant la journée, suivant un parcours systématique permettant une collecte représentative et excluant les bordures. Le traitement des échantillons s'effectue par séchage, broyage et tamisage pour l'obtention de poudres demi-fines $(1 \mathrm{~mm})$. L'extraction des cannabinoïdes est effectuée par macération de $100 \mathrm{mg}$ de poudre dans $5 \mathrm{~mL}$ de solution d'extraction : $35 \mathrm{mg}$ de standard interne le squalène (Sigma réf. S3626) dans 100 mL d'hexane (J.T. Baker réf. 8044). Les analyses chimiques sont menées sur un GCFID Agilent 6890N, équipé d'une colonne apolaire (HP-5, $5 \%$ phényl-méthyl-siloxane, $30 \mathrm{~m} \times 0,32 \mathrm{~mm} \times 0,22 \mu \mathrm{m})$. Les injections sont réalisées en mode division $25 / \mathrm{L}$, dans les conditions de température suivantes : $T_{\text {four }}: 200{ }^{\circ} \mathrm{C}$ - rampe de $10^{\circ} \mathrm{C} /$ min jusqu'à $280{ }^{\circ} \mathrm{C}$ isotherme $10 \mathrm{~min}, T_{\text {inj }}: 280{ }^{\circ} \mathrm{C}$, $T_{\text {det }}: 300^{\circ} \mathrm{C}$.

La courbe d'étalonnage (figure 2) est établie à l'aide de 6 concentrations 0,$005 ; 0,01 ; 0,02 ; 0,04 ; 0,1 ; 0,2$ et $0,4 \mathrm{mg} / \mathrm{mL}$ de standard $\Delta-9$-THC (LGC Promochem réf. T-005) en so- lution d'extraction. Trois prises d'essais sont considérées par échantillon et le résultat final correspond à la moyenne obtenue d'une double analyse de ces trois prises d'essais.

\section{Résultats et discussion}

Comparativement aux cultures européennes, le cycle de croissance de la plante a été plus rapide dans les quatre régions testées puisqu'au bout de 2 mois, le stade de fin de floraison a été atteint dans les régions d'Agadir, Béni-Mellal et Allal Tazi et au bout de 2,5 mois pour la région de Sefrou. Ce comportement peut s'expliquer par la photopériode caractérisant ces régions, particulièrement favorable au développement de la plante qui semble ne pas être très affecté par les propriétés intrinsèques de ces régions (altitude, nature du sol, pluviométrie, etc.).

\subsection{Aspects agronomiques}

Les rendements en matière sèche (tonne/hectare) obtenus pour les trois variétés sont très variables selon les sites. Le niveau de production varie entre $24,5 \mathrm{t} / \mathrm{h}$ réalisé par la variété Futura 75 à Béni-Mellal et 4,47 t/ha réalisé par Santhica 27 à Sefrou (tableau IV).

Au niveau de tous les sites, la variété Futura 75 est la plus performante, suivie de la variété Epsilon 68 et en dernier la variété Santhica 27. Futura 75 germe mieux que les autres variétés sur tout type de sol argileux (Béni-Mellal, Allal Tazi), ou caillouteux (Sefrou) ou encore sablonneux (Agadir).

Le site de Béni-Mellal est le plus favorable des quatre sites, avec une production moyenne de toutes les variétés de 20,72 t/ha. Il est suivi de Allal Tazi, où la production moyenne de toutes les variétés est de 10,87 t/ha, Les conditions pédologiques et climatiques de Béni-Mellal semblent donc les mieux appropriées.

Les rendements réalisés à Béni-Mellal représentent $85 \%$ des rendements potentiels mesurés en France (FNCP, 2008) pour la variété Futura 75 (28,5 t/ha), $63 \%$ pour la variété Santhica $27(25,75$ t/ha) et $80 \%$ pour la variété Epsilon 68 (26,70 t/ha).

Les hauteurs des plantes sont variables de 1,14 à 2,27 m, avec les meilleures performances enregistrées dans la région Béni-Mellal (2,27 m). La variété Futura 75 a généré dans les quatre régions les plus hautes plantes (tableau IV).

Les densités obtenues sont moyennes, variables entre 88 et 30 (plantes $/ \mathrm{m}^{2}$ ) mais peuvent être améliorées par optimisation 


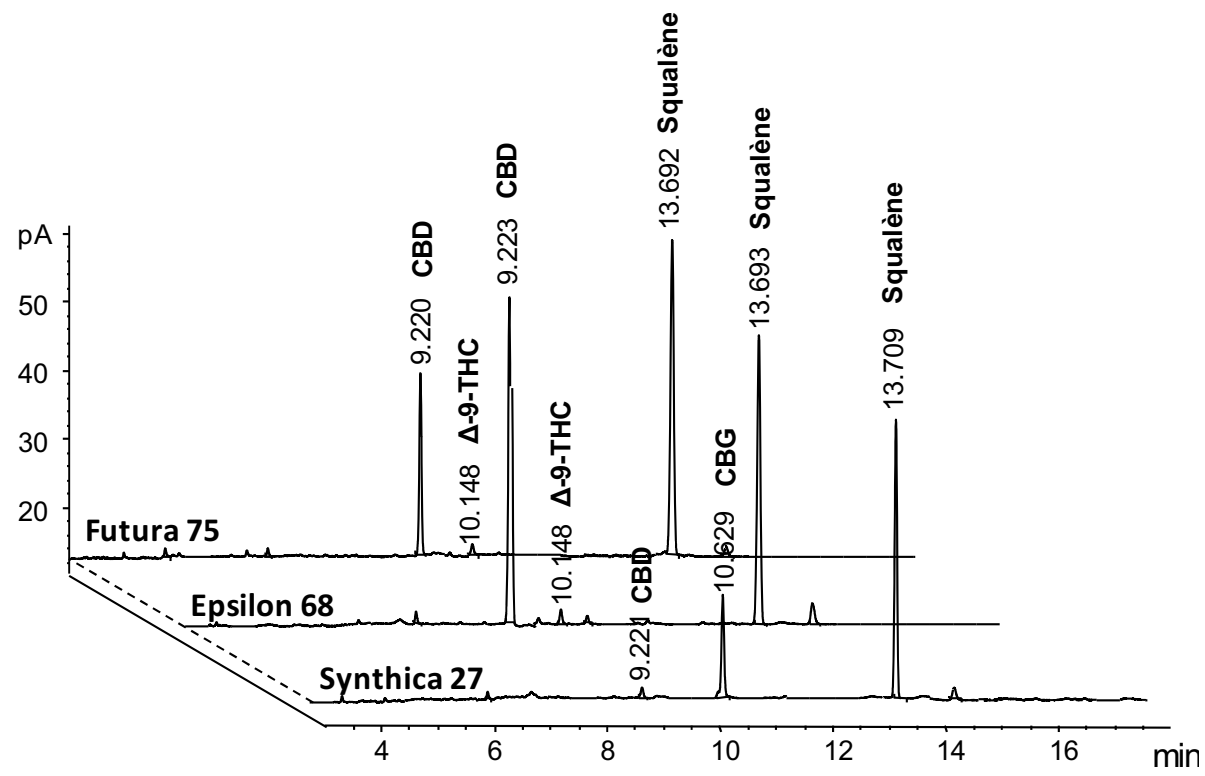

Fig. 3. Profils chromatographiques FID des variétés Santhica 27, Epsilon 68 et Futura 75.

Tableau V. Teneurs en $\Delta$-9-THC pour les trois variétés Santhica 27, Epsilon 68 et Futura 75.

\begin{tabular}{|c|c|c|c|c|c|}
\hline \multirow{2}{*}{ Variété } & \multicolumn{5}{|c|}{ Teneur en \% $\Delta$-9-THC } \\
\cline { 2 - 6 } & Sefrou & Béni-Mellal & Allal Tazi & Agadir & Moyenne \\
\hline Santhica 27 & ND & ND & ND & ND & ND \\
\hline Epsilon 68 & 0,013 & 0,024 & 0,026 & 0,027 & 0,022 \\
\hline Futura 75 & 0,023 & 0,021 & 0,025 & 0,035 & 0,026 \\
\hline
\end{tabular}

des conditions de semis et par le travail de la terre. La Futura 75 se distingue nettement par ses meilleures densités dans les quatre régions. Dans les conditions de températures et d'ensoleillement locales, la densité peut être revue à la hausse en couvrant les besoins correspondants en eau et éléments fertilisants pour assurer des rendements plus élevés.

\subsection{Les teneurs en $\Delta-9-\mathrm{THC}$}

Les teneurs moyennes en $\triangle$-9-THC déterminées pour les 12 échantillons de poudres issus des variétés Epsilon 68, Futura 75 et Santhica 27, sont respectivement de 0,022\%, $0,026 \%$, et $<0,005 \%$; elles se situent toutes nettement en dessous de la norme européenne fixée à 0,2\% (tableau V).

Ainsi, pour les quatre sites, la variété Santhica 27 est considérée exempte de $\Delta-9$-THC $(<0,001 \%)$, et présente des teneurs notables en CBG (figure 3 ). Toutefois, l'analyse en GC/MS mode SIM $(314,299,231)$ ou en LCMSMS (MRM $193.0 ; 123.0 ; 107.2 ; 93.0$ ) (figures 4 et 5 ) a décelé au sein de cette variété de faibles traces de $\Delta$-9-THC. Les deux autres variétés, Epsilon 68 et Futura 75, présentent des teneurs en $\triangle$-9-THC situées respectivement dans les intervalles $(0,013 \%-0,027 \%)$ et $(0,021 \%-0,035 \%)$ à côté de proportions plus importantes en CBD (figure 3).

De manière générale, il a été noté que les teneurs en $\Delta-9$ THC enregistrées pour les sites d'Agadir sont légèrement plus élevées comparativement aux autres régions et ce, particulièrement pour la variété Futura 75.

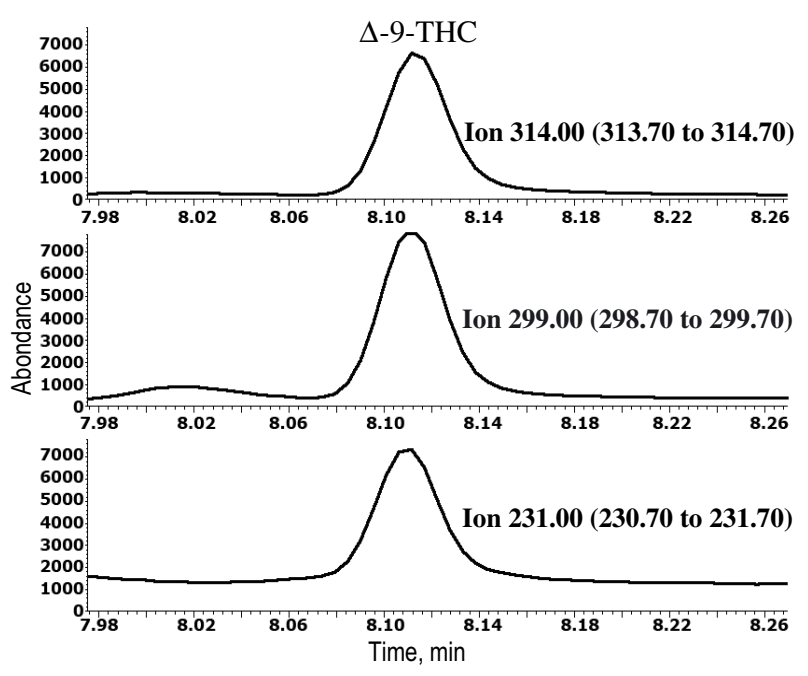

Fig. 4. Mise en évidence de traces du $\Delta-9-\mathrm{THC}$ au sein de la variété Santhica 27 par analyse GC/MS en mode SIM.

\subsection{Le rapport $\alpha=\Delta-9-\mathrm{THC} / \mathrm{CBD}$}

La variété Santhica 27 étant dépourvue de $\Delta$-9-THC $(<0,005 \%)$, le rapport $\alpha$ correspondant est donc nul. Pour les variétés Futura 75 et Epsilon 68, leur rapport évalué respectivement à 0,052 et 0,056 , est du même ordre de grandeur que celui obtenu habituellement pour les cultures de chanvre à fibres et reste nettement inférieur à 0,2 (tableau VI). 
Tableau VI. Rapports $\alpha$ des deux variétés Futura 75 et Epsilon 68.

\begin{tabular}{|c|c|c|c|c|c|}
\hline Variété & Paramètre & Agadir & Béni-Mellal & Allal Tazi & Sefrou \\
\hline \multirow{4}{*}{ Futura 75 } & $\Delta$-9-THC* & 1,45 & 1,47 & 1,3 & 1,58 \\
\cline { 2 - 6 } & CBD $^{*}$ & 27,88 & 28,47 & 24,27 & 30,47 \\
\cline { 2 - 6 } & $\alpha=\Delta-9-$ THC/CBD & 0,052 & 0,052 & 0,053 & 0,052 \\
\cline { 2 - 6 } & $\alpha$ moyenne & \multicolumn{4}{|c|}{0,052} \\
\hline \multirow{4}{*}{ Epsilon 68 } & $\Delta$-9-THC* & 0,89 & 1,23 & 1,10 & 1,25 \\
\cline { 2 - 6 } & CBD* $^{*}$ & 13,98 & 21,18 & 20,55 & 24,35 \\
\cline { 2 - 6 } & $\alpha=\Delta$-9-THC/CBD & 0,063 & \multicolumn{5}{|c|}{0,058} & 0,053 & 0,051 \\
\cline { 2 - 6 } & $\alpha$ moyenne & \multicolumn{4}{|c|}{0,056} \\
\hline
\end{tabular}

*Aire du pic chromatographique.

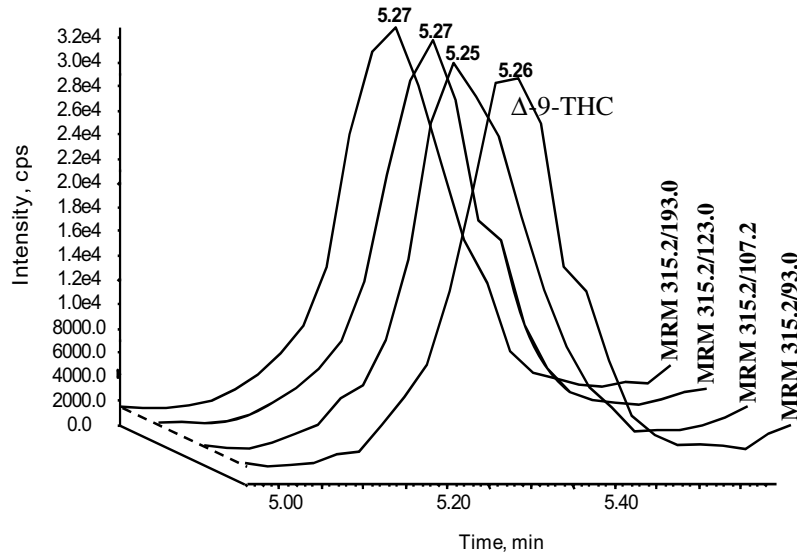

Fig. 5. Mise en évidence de traces du $\Delta-9-\mathrm{THC}$ au sein de la variété Santhica 27 par analyse LCMSMS.

\section{Conclusion}

Cette première expérimentation de la culture de chanvre à fibres au Maroc a concerné trois variétés Santhica 27, Epsilon 68 et Futura 75 testées dans quatre régions différentes. Le stade de maturation des trois variétés a été atteint au bout de 2,5 mois et ce, quelle que soit la nature caillouteuse, argileuse ou sablonneuse du sol. Les rendements obtenus en matière sèche, densité et hauteur des plantes sont légèrement inférieurs à ceux observés notamment en Europe mais peuvent être améliorés par un meilleur contrôle des conditions de semis, des besoins en eau, le travail de la terre et l'apport en fertilisants. Les meilleurs résultats sont obtenus avec la variété Futura 75 dans les quatre sites, suivie de Epsilon 68 et de Santhica 27.

Le contrôle de la teneur en $\Delta-9$-THC selon la procédure B du règlement $\mathrm{CE}$ n'a enregistré aucun dépassement de la limite de $0,2 \%$. De même, le rapport $\alpha(\Delta-9-\mathrm{THC} / \mathrm{CBD})$ déterminé pour les deux variétés Epsilon 68 et Futura 75, est de même ordre de grandeur que les valeurs classiquement obtenues pour ces cultures.

Ces résultats indiqueraient que les conditions environnementales n'ont pas influé significativement sur le comportement des trois variétés testées dans quatre régions différentes par leurs conditions climatiques, situations géographiques et caractéristiques pédologiques.
Ces essais préliminaires étaient nécessaires antérieurement à toute mise en place d'instances administratives qui seront chargées de trouver un cadre réglementaire fixant les aspects techniques et économiques, notamment la procédure nationale de contrôle et de surveillance des cultures, délivrance des autorisations d'importation des semences ou d'exportation de chanvre industriel et de ses dérivés. De même, l'arsenal juridique devra être renforcé pour décourager toute tentative mal intentionnée de plantation de chanvre type drogue à la place de la fibre.

Remerciements. J'exprime ma gratitude au Pr. Gilbert Fournier pour les discussions techniques que j'ai pu avoir avec lui à ce sujet et adresse mes remerciements à la Fédération Nationale des Producteurs de Chanvre FNPC, qui nous a fourni les semences nécessaires à cette expérimentation.

Conflit d'intérêt. Les auteurs déclarent ne pas avoir de conflit d'intérêt.

\section{Références}

1. Document consulté sur le site http://drigues.suite101.fr/article. cfm, le $1^{\text {er }}$ novembre 2010, Marie-Paule Hom. Les différentes utilisations du cannabis : Chanvre indien, chanvre cultivé : leurs principales applications.

2. Fournier G, Bausset J, Maciuk A, Beherec O, Desvals M, Bertucelli S. Bilan de huit années de contrôle des cultures de chanvre industriel. Ann Toxicol. Anal. 2008; 20(4): 217-221.

3. Document consulté sur le site http://www.naturalfibres2009.org/ fr/index.html, le $1^{\text {er }}$ novembre 2010.

4. Document consulté sur le site http://www.interchanvre.com/ ressources.php, le $1^{\text {er }}$ novembre 2010.

5. Fournier G. Une nouvelle souche de chanvre à fibres sans cannabinoïdes. Ann Toxicol Anal. 2005; 17(2): 109-111.

6. Journal officiel des Communautés Européennes - Procédure B du Règlement CE N ${ }^{\circ}$ 1177/2000 (Arrêté du 24 février 2004), fixant conditions, échantillonnage, conservation, séchage, broyage et analyse - Annexe $\mathrm{C}$ du règlement (CE) $\mathrm{N}^{\circ} 1177 / 2000$ de la commission du 31 mai 2000.

7. Fournier G, Béhérec $\mathrm{O}$, Bertucelli S. Intérêt du rapport $\Delta-9$ THC/CBD dans le contrôle des cultures de chanvre industriel. Ann Toxicol Anal. 2003; 15(4): 250-259.

8. Fournier G, Bausset J, Beherec O, Desvals M, Bertucelli S. La simplification du contrôle des cultures de chanvre industriel est possible. Ann Toxicol Anal. 2007; 19(3): 201-209. 